\title{
A Novel Compact Triple Bandpass Filter using Microstrip Resonator
}

\author{
Sara Jafarpoor \\ Department of Communication, \\ Bushehr Branch, \\ Islamic Azad University \\ Bushehr, Iran.
}

\author{
Alireza Mallahzadeh \\ Department of \\ Communication,khalij fars \\ University,Bushehr,Iran
}

\author{
Sahar Mardani \\ Ghahfarokhi \\ Department of Communication, \\ Bushehr Branch, \\ Islamic Azad University \\ Bushehr, Iran.
}

\begin{abstract}
In this paper, a compact triple-narrowband bandpass filter is designed and proposed using microstrip resonators. The proposed triple-band BPF consists of two half-wave resonators in combination with a simple anti-parallel coupled lines which realizes a triple-band BPF with a size reduction about $42 \%$ in comparison with the conventional one. Additionally, transmission zeroes of the proposed structure guarantee the sharpness the skirts of scattering parameters of triple-band BPF. The frequency response of the filter is simulated using an EM simulation tool.
\end{abstract}

\section{Keywords}

Anti-parallel coupled lines, half-wave resonator, interdigital capacitor, substrate, triple-narrowband bandpass filter

\section{INTRODUCTION}

Current advancements in modern wireless communication systems demonstrate the needs for smaller and low-cost microwave components. In this connection, several communication systems such as personal ones operate in multi bands which necessary narrow-band kinds of such components. Dual/triple-narrowband bandpass filters (BPF) are one of the most popular components increasingly investigated in the most recent studies. Many triple-band BPFs have been introduced by different topologies in several investigations: however, they still occupy a relatively large area on their substrates and often with complicated structures [1-10]. For instance, a triple-band BPF has been proposed in [3] using asymmetric coupled lines where it has been designed using multi-layered technology with a size about $64 \times 38 \mathrm{~mm}^{2}$ on its substrate.

Moreover, a triple-band bandpass filter has recently been developed using stepped-impedance resonators (SIRs) which provide dual-band bandpass one, though its size is about 533 $\mathrm{mm}^{2}$ that is still relatively large on circuit boards [4].

Furthermore, another triple-band BPF has been analyzed and introduced in [5] by an active size around $24 \times 19.5 \mathrm{~mm}^{2}$.

Besides, several techniques based on microstrip open and closed loop resonators have been discussed on different studies [6-7]. Although, they have simple design procedure, they occupy large area on their substrate.

Here is a novel compact triple-band BPF designed and simulated using the combination of half-wave resonators and anti-parallel coupled lines. The proposed BPF realizes a

compact triple-band one. It is designed and simulated on a 31mill-thickness substrate with a constant dielectric of 2.2 .

\section{DESIGN PROCEDURE}

As stated, this paper introduces a new kind of triple-band BPF developed using a combination of half-wave resonators and antiparallel coupled lines. Fig.1 shows the schematic diagram of this proposed triple-band BPF.

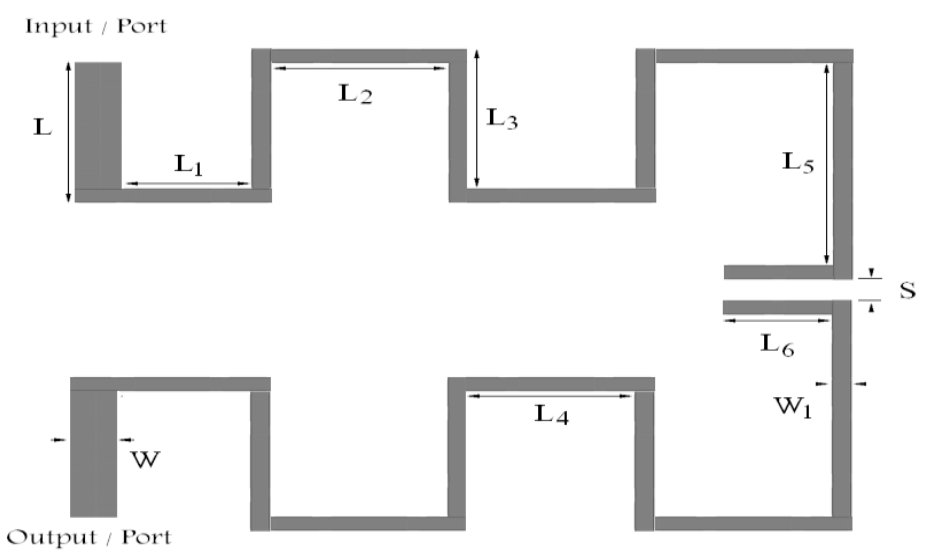

Fig 1: The structure of the conventional microstrip open-loop resonators.

The proposed layout of the triple band band-pass filter is simulated on a 31-mill-thickness substrate with a constant dielectric of 2.2 and the indicated parameters on the structure are optimized using a full-wave simulator tool (ADS) where the table 1 gives these optimized parameters.

Table 1 : Optimized parameters of the proposed topology shown in Fig 1.

\begin{tabular}{|c|c|c|c|c|c|}
\hline Parameter & $\mathrm{L}$ & $\mathrm{L}_{1}$ & $\mathrm{~L}_{2}$ & $\mathrm{~L}_{3}$ & $\mathrm{~L}_{4}$ \\
\hline Value & 1.07 & 2 & 5 & 2.14 & 2 \\
\hline Parameter & $\mathrm{L}_{5}$ & $\mathrm{~L}_{6}$ & $\mathrm{~S}$ & $\mathrm{~W}$ & $\mathrm{~W}_{1}$ \\
\hline Value & 5 & 3 & 0.05 & 0.5 & 0.07 \\
\hline
\end{tabular}

After optimization of the parameters, the performance of the proposed triple-band band-pass filter is simulated using ADS software where Fig.2 shows the frequency response of the filter in terms of the insertion and return losses, respectively. 
S21

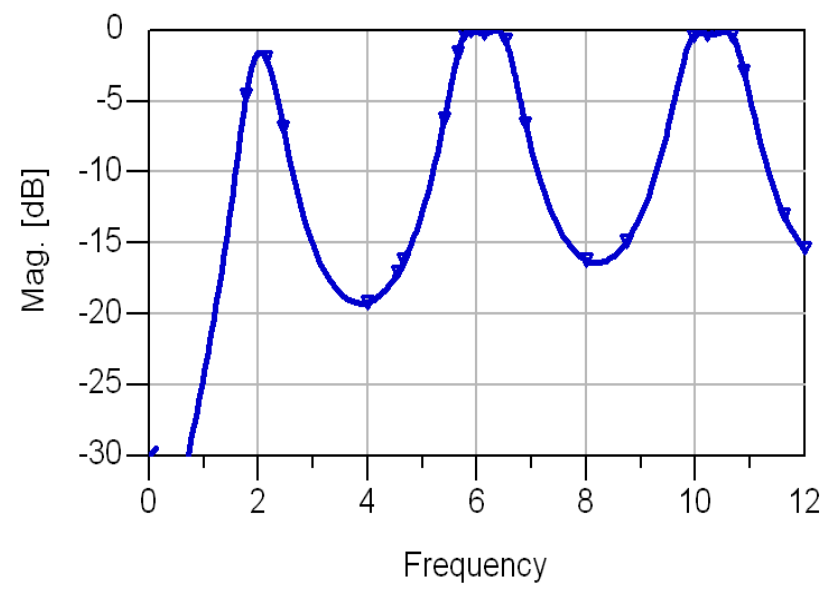

(a)

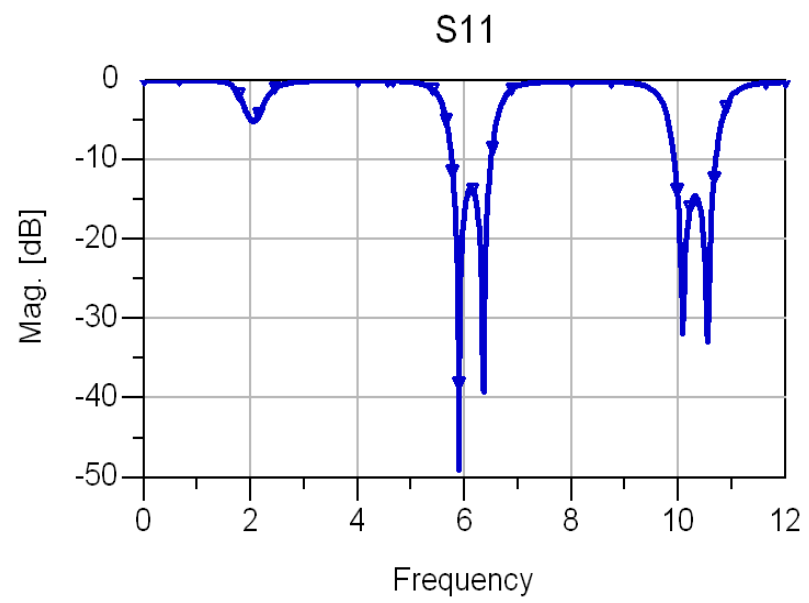

(b)

Fig 2: The frequency response of the proposed triple-band bandpass filter in terms of (a) insertion loss (b) return loss.

Observing the performance of the proposed structure, this novel filter provides three different bands located at $2 \mathrm{GHz}, 6.1 \mathrm{GHz}$ and $10.4 \mathrm{GHz}$.

Additionally, the performance of the proposed filter is simulated in terms of current distribution where Fig. 3 shows this parameter at the third resonance frequency.

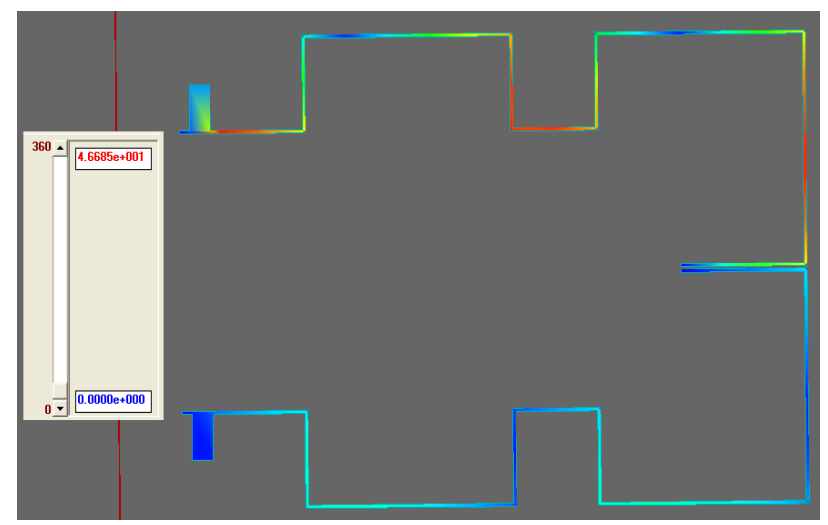

Fig 3: The current distribution of the proposed triple-band band-pass filter at the third resonance frequency.
The main disadvantage of the proposed filter is the high insertion and return losses in the first band of the frequency response which is tried to improved these by upgrading the structure of the filter by incorporating of the interdigital capacitor instead of the anti-parallel coupled lines.

\section{SIMULATION RESULTS}

According to the given specifications in the previous sections, to improve the insertion and return loss of the proposed filter, a compact triple-band bandpass filter can be designed and simulated using a novel combination of half-wave resonators and interdigital capacitor. The proposed topology shown in Fig.1 is thus implemented by interdigital capacitor instead of the antiparallel coupled lines and simulated using an EM simulator tool (ADS). A fine tuning process is carried out to optimize the dimensions to have a triple-band bandpass filter. Table 2 gives the optimized parameters of the interdigital capacitor.

Table 2 : The optimized parameters of the interdigital capacitor.

\begin{tabular}{|c|c|c|c|c|c|}
\hline Parameter & $\mathrm{L}$ & $\mathrm{W}$ & $\mathrm{W}_{1}$ & $\mathrm{G}$ & $\mathrm{S}$ \\
\hline Value & 2 & 0.1 & 0.6 & 0.12 & 0.12 \\
\hline
\end{tabular}

Fig. 4 shows the simulated frequency response of the proposed triple-band bandpass filter in terms of the insertion and return losses, respectively.

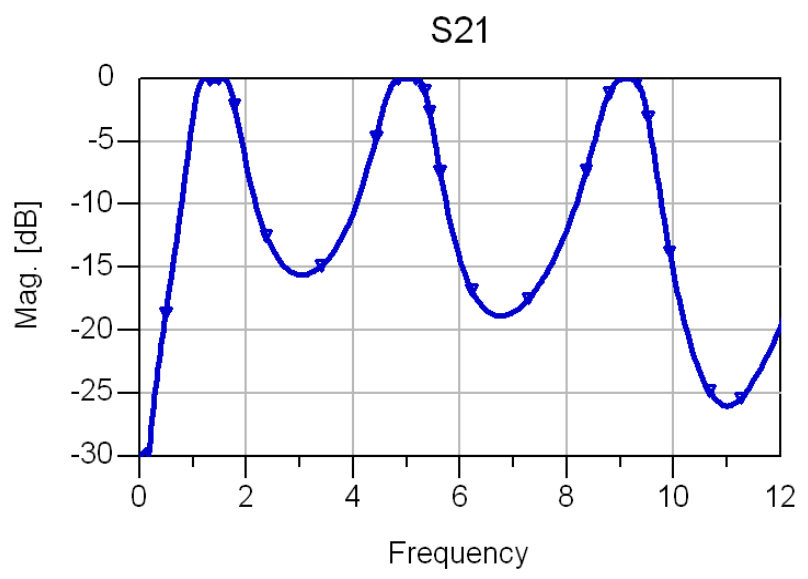

(a)

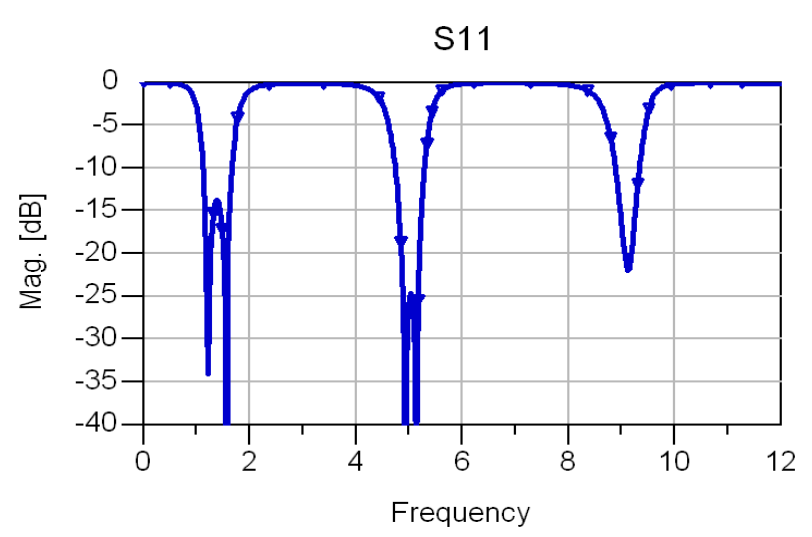

(b)

Fig 4: The frequency response of the proposed triple-band bandpass filter in terms of (a) insertion loss (b) return loss. 
Comparing the frequency responses of the filter using antiparallel coupled lines - given in Fig.2- and the one with interdigital capacitor - given in Fig.4-, the use of interdigital capacitor obviously improves the insertion and return losses of the filter in the first band.

Simulated central frequencies are $1765 \mathrm{MHz}, 5030 \mathrm{MHz}$ and $9025 \mathrm{MHz}$ with 3-dB bandwidths of $6.8 \%, 5.2 \%$ and $3.9 \%$, respectively, making this compact triple-band BPF very attractive and suitable for applications in the mobile and personal communication systems (PCS's). Besides, the maximum insertion losses are reported to be $0.7 \mathrm{~dB}, 0.4 \mathrm{~dB}$ and $1 \mathrm{~dB}$ in the first, second and third bands, respectively. The return losses for these three bands are better than $-14 \mathrm{~dB},-25 \mathrm{~dB}$ and $21 \mathrm{~dB}$, respectively.

\section{CONCLUSION}

In this paper, a miniaturized triple-narrowband bandpass filter has been designed and proposed using microstrip resonators. The proposed triple-band BPF consists of two half-wave resonators in combination with a simple anti-parallel coupled lines which realizes a triple-band BPF with a size reduction about $42 \%$ in comparison with the conventional one. Additionally, transmission zeroes of the proposed structure guarantee the sharpness the skirts of scattering parameters of triple-band BPF. The frequency response of the filter has been simulated using an EM simulation tool.

\section{REFERENCES}

[1] Xuehui Guan, Zhewang Ma, and Peng Cai "A novel tripleband microstrip bandpass filter for wireless communicaton," Microwave and Optical Technology Letter, Vol. 51, No. 6, June 2009.

[2] Ching-Her Lee,, Chung-I G. Hsu, and Yu-Cheng Chen "Compact tri-band BPF desing using spur-line-loaded trisection SIRS,"Microwave and Optical Technology Letter, Vol. 51, No. 14, April 2009.
[3] Hui Zhao and Tie Jun Cui "Desing of triple-band filters by using asymmetric parallel coupled lines," Microwave and Optical Technology Letter, Vol. 50, No. 8, Aug. 2008.

[4] K. Rambabu, Adrian E. C. Tan, Michael Y. W. Chia, "PRrinted-circuit filters for wireless doul-and triple-band application," Microwave and Optical Technology Letter, Vol. 50, No. 6, June. 2008.

[5] Cheng-Yuan Kung, Yin-Chung Chen, Cheng-Fu Yang, and Chih-Yu Huang "TRriple-band parallel couplid microstrip bandpass filter with doul coupled length input/output," Microwave and Optical Technology Letter, Vol. 51, No. 4, April 2009.

[6] C.-H. Lee, C.-I.G. Hsu, and H.-K. Jhuang, Design of a new tri-band microstrip BPF using combined quarter-wavelength SIRs, IEEE Microwave Wireless Compon Lett 16 (2006), $594-596$.

[7] Chung-I G. Hsu, Ching-Her Lee, and Yi-Huan Hsieh"TriBand Bandpass Filter with Sharp Passband Skirts Designed Using Tri-Section SIRs," IEEE Microwave Wireless Compon Lett 18 (2008).

[8] Mo, S.-G., Z.-Y. Yu, and L. Zhang, IDesign of triple-mode bandpass filter using improved hexagonal loop resonator," Progress In Electromagnetics Research, Vol. 96, 117-125, 2009.

[9] Lin, X.-M., IDesign of compact tri-band bandpass filter using , $\lambda / 4$ and stub-loaded resonators," Journal of Electromagnetic Waves and Applications, Vol. 24, No. 14, 2029-2035, 2010.

[10] Goudos, S. K., Z. D. Zaharis, and T. Yioultsis, "Application of a differential evolution algorithm with strategy adaptation to the design of multi-band microwave filters for wireless communications," Progress In Electromagnetics Research, Vol. 109, 123-137,2010. 